\title{
Characterization of cholesterol metabolism in Sertoli cells and spermatogenesis (Review)
}

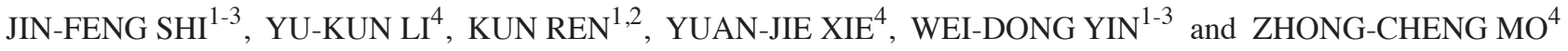 \\ ${ }^{1}$ Institute of Cardiovascular Disease, Medical School, University of South China; ${ }^{2}$ Key Laboratory for Arteriosclerology \\ of Hunan Province; ${ }^{3}$ Hunan Province Cooperative Innovation Center for Molecular Target New Drug Study; ${ }^{4}$ Department \\ of Histology and Embryology, Medical School, University of South China, Hengyang, Hunan 421001, P.R. China
}

Received March 28, 2017; Accepted August 31, 2017

DOI: $10.3892 / \mathrm{mmr} .2017 .8000$

\begin{abstract}
The Sertoli cell, which is the supporting cell of spermatogenesis, has an important role in the endocrine and paracrine control of spermatogenesis. Functionally, it provides the cells of the seminiferous epithelium with nutrition, conveys mature spermatids to the lumen of seminiferous tubules, secretes androgen-binding protein and interacts with endocrine Leydig cells. In addition, the levels of cholesterol, as well as its intermediates, vary greatly between nongonadal tissues and the male reproductive system. Throughout spermatogenesis, a dynamic and constant alteration in the membrane lipid composition of Sertoli cells occurs. In several mammalian species, testis meiosis-activating sterol and desmosterol, as well as other cholesterol precursors, accumulate in the testes and spermatozoa. In addition, certain cholesterogenic genes exhibit stage-specific expression patterns during spermatogenesis, including the cytochrome P450 enzyme lanosterol $14 \alpha$-demethylase. Inconsistency in the patterns of gene expression during spermatogenesis indicates a cell-type specific and complex temporary modulation of lipids and cholesterol, which also implicates the dynamic interactions between Sertoli cells
\end{abstract}

Correspondence to: Dr Zhong-Cheng Mo, Department of Histology and Embryology, Medical School, University of South China, 28W Changsheng Road, Hengyang, Hunan 421001, P.R. China E-mail: zhchmo@hotmail.com

Professor Wei-Dong Yin, Institute of Cardiovascular Disease, Medical School, University of South China, 28W Changsheng Road, Hengyang, Hunan 421001, P.R. China

E-mail: wdy20042004@126.com

Abbreviations: ABP, androgen-binding protein; ABCA1, ATP-binding cassette subfamily A member 1; LDL, low-density lipoprotein; HDL, high-density lipoprotein; AMH, anti-Müllerian hormone; FSH, follicle-stimulating hormone; SR-BI, scavenger receptor class B member I; RCT, reverse cholesterol transport; CE, cholesterol esters; LXR, liver X receptor; AS, atherosclerosis; RXR, retinoid $X$ receptor; SREBP-1c, sterol regulatory element-binding protein-1c; PS, phosphatidylserine

Key words: cholesterol metabolism, Sertoli cells, spermatogenesis and germ cells. Furthermore, in the female reproductive tract and during epididymal transit, which is a prerequisite for valid fertilization, the modulation of cholesterol occurring in spermatozoal membranes further indicates the functional importance of sterol compounds in spermatogenesis. However, the exact role of cholesterol metabolism in Sertoli cells in sperm production is unknown. The present review article describes the progress made in the research regarding the characteristics of the Sertoli cell, particularly the regulation of its cholesterol metabolism during spermatogenesis.

\section{Contents}

1. Introduction

2. The role of Sertoli cells in spermatogenesis

3. The role of cholesterol in Sertolicells during spermatogenesis

4. Conclusions and perspectives

\section{Introduction}

Subfertility refers to the failure of conception following unprotected intercourse for at least one year. Many couples suffer from subfertility problems worldwide, with estimates ranging from $10-20 \%$. Of the total infertility cases, $\sim 50 \%$ are attributed to male infertility (1). Dysfunctional spermatogenesis is among the leading causes of male subfertility, and assessment of the quality of spermatogenesis is crucial for the evaluation and treatment of subfertility in men. A previous study reported that the Sertoli cell promotes sperm production through various functions, including nurturing seminiferous epithelial cells, transporting spermatids and secreting androgen-binding protein (ABP) (2). In addition, studies have also identified that cholesterol metabolism-associated genes, including ATP-binding cassette (ABC) subfamily A member 1 (ABCA1)/G5 $(3,4)$, apolipoprotein B (5), cystic fibrosis transmembrane conductance regulator (6) and cAMP-responsive element modulator (7), are essential for normal male fertility. Sertoli cells exert phagocytic functions similar to macrophages and possess the ability to efflux excess lipids and cholesterols after engulfing membrane-rich structures. By means of the basement membrane, Sertoli cells are isolated from interstitial capillaries, which blocks the passage of low density lipoprotein 
(LDL) but permits the entry of high density lipoprotein (HDL) to the seminiferous tubules (8). The molecular pathways responsible for the regulation of lipid exchange between the periphery and testes remain unclear, but are of great interest due to the critical role of cholesterol in spermatogenesis and steroidogenesis. Furthermore, Leydig and Sertoli cells produce reproductive steroid hormones. Leydig cells secrete a number of different types of androgens, including dihydrotestosterone and testosterone, which modulate the development and maturation of spermatozoa (9). The uptake of testosterone by Sertoli cells leads to its conversion into dihydrotestosterone and estradiol. Leydig cells obtain cholesterol either via de novo synthesis pathways or through the uptake of cholesterol from HDL (10). In addition, although Sertoli cells use acetate to synthesize cholesterol, their primary source of cholesterol is from HDL entry from the plasma or androgens that are transported from Leydig cells $(8,11,12)$. The following review is a concise overview of the progress of research concerning cholesterol metabolism in Sertoli cells in addition to its role in spermatogenesis.

\section{The role of Sertoli cells in spermatogenesis}

The Sertoli cell, which was initially identified by Enrico Sertoli in 1865 , performs a critical role during the process of spermatogenesis. Sertoli cells are recognized as 'nurse cells' that are responsible for extending nutritional and energy support to the development of germ cells. It has been widely demonstrated that germ cells require a sufficient level of energy resources, otherwise they decay and enter apoptosis $(13,14)$. The development of germ cells requires specific metabolic substrates, such as lactate, which is used as a substrate for ATP production (15). Through the secretion of metabolic intermediates or nutrients, including carbohydrates, lipids, vitamins, metal ions and amino acids, Sertoli cells provide the nutritional requirements of germ cells $(16,17)$.

Additionally, through characteristic zones of cellular membrane tight junctions, Sertoli cells form connections with one another and divide the germinal epithelium into an adluminal and a basal compartment; the blood-testis barrier of the testis is also formed by these tight junctions. After passing this barrier, the germ cells are protected from extraneous substance diffusion in the adluminal compartment during spermatogenesis (2). Therefore, the blood-testis barrier acts as a multifunctional boundary between haploid and diploid germ cells, in addition to separating testicular tissue from blood. Furthermore, a functional Sertoli cell provides energy, differentiation factors and adequate mitogens to the developing germ cells, and prevents them from harm that may result from the host's own immune system (18). Sertoli cells also produce various types of enzymes, growth factors and hormones, including plasminogen activator, ABP, ceruloplasmin, insulin-like growth factor, transforming growth factors $\alpha$ and $\beta$, anti-Müllerian hormone (AMH) and inhibin B $(2,18)$.

Sertoli cells express the sex-determining region $\mathrm{Y}$ gene, and thereby decide the male sex of the gonad. By producing AMH, Sertoli cells also suppress the development of internal female genitalia. Together with the peritubular cells, these cells are critical for the formation of the testis cords. The immature Sertoli cell varies greatly from the mature one in terms of biochemical activity and morphology. During puberty, the Sertoli cells are stretched and begin to form connections through tight junctions. Furthermore, they secrete seminiferous fluid, transforming testis cords into seminiferous tubules. The mature differentiated Sertoli cell alters its protein expression pattern, producing various factors, including the inflammatory cytokine interleukin- $1 \alpha$ and transferrin. As puberty develops, despite the consecutive division of mature Sertoli cells, their proliferative activity is reduced. After the formation of tight junctions, Sertoli cells exhibit no proliferative capacity (18). Follicle-stimulating hormone (FSH) serves as the predominant endocrine factor responsible for the regulation of Sertoli cell function. In the testes, the Sertoli cell exclusively expresses FSH receptors, which is required for the appropriate proliferation of Sertoli cells. Spermatogenesis relies on an appropriate intratesticular level of testosterone. Furthermore, Sertoli cells, but not germ cells, express the androgen receptor, indicating that Sertoli cells mediate the effects of androgen on the seminiferous epithelium. The androgen receptor is necessary for the correct functioning of the blood-testis barrier, as well as for the normal development of germ cells, and its expression on Sertoli cells was reported to be amplified throughout the maturation process of germ cells (18). Therefore, Sertoli cells have crucial roles in the autocrine and/or paracrine regulation of spermatogenesis (2).

\section{The role of cholesterol in Sertoli cells during spermatogenesis}

Lipids and cholesterols are essential for spermatogenesis as they serve as 'fuel' for Sertoli cells and are crucial for the membrane remodeling of developing germ cells. The biosynthesis pathway of cholesterol comprises a succession of enzymatic reactions implicating diverse intermediates. Among them, meiosis-activating sterols were reported to have strong meiosis-activating potency through screening of naturally occurring compounds. In addition, various factors are responsible for the regulation of cholesterol content in Sertoli cells, including ABCA1 and scavenger receptor class B member 1 (SR-BI) $(8,9,18,19)$.

The transport of cholesterol in Sertoli cells. A large proportion of nutrients, such as lipids, used for spermatogenesis are provided by supporting Sertoli cells. Sertoli cells have the ability to synthesize cholesterol using acetate in vitro (19), however, it has been demonstrated that this source of cholesterol is insufficient for steroidogenesis in vivo. As the amount of cholesterol required for spermatogenesis far exceeds the capacity of Sertoli cells biosynthesis, specialized cholesterol transporters such as SR-BI facilitate the uptake of cholesterol from the blood (20). Research has revealed that, at least in rodents, HDL serves as the primary source of cholesterol for Sertoli cells (21), which may be further supported by the observations that the basal membrane segregating seminiferous tubules from blood capillaries blocks the entry of LDL but permits HDL entry $(8,21)$. In rats, Sertoli cells obtain cholesterol from HDL primarily through apolipoprotein E-dependent pathways (22). In addition, Sertoli cells may also obtain cholesterol from phagocytosed apoptotic germ cells and lipid-rich remnant recycling (22). To eliminate toxicity, 
surplus cholesterol undergoes esterification and storage in lipid droplets, which is instrumental in the maintenance of cholesterol equilibrium. Lipid droplets are highly mobile and dynamic structures, and are reported to interact with cell junctions and organelles via transient or stable surface exchange, ensuring the effective synthesis, uptake, usage and elimination of cholesterol (23). Furthermore, Sertoli cells also maintain cholesterol homeostasis through reverse cholesterol transport (RCT). RCT involves various procedures that enable the efflux of excess cholesterol to HDL and promote the transport of cholesterol from non-hepatic peripheral tissue back to the liver via the plasma to maintain cholesterol homeostasis, which primarily relies on the ABCA1 cholesterol transporters (3). The excessive accumulation of cholesterol esters (CE) in Sertoli cells leads to disruption of cholesterol homeostasis, ultimately resulting in complete subfertility or infertility. This phenomenon was demonstrated in various knockout mouse models that are deficient in functional nuclear receptors, such as retinoid X receptor (RXR) $(24,25)$, and also in ABCA1 knockout mice (3). As ABCA1 is an established liver $\mathrm{X}$ receptor (LXR) target gene, in double knockout mice (LXR $\alpha / \beta^{--}$), the deficiency of ABCA1 decreases the cholesterol efflux from Sertoli cells and results in the accumulation of CE $(3,24)$. Therefore, Sertoli cells modulate every step of cholesterol metabolism, including cholesterol uptake, efflux, storage and recycling, which indicates that Sertoli cells should be a focus in the interpretation of spermatogenesis. Fig. 1 is a graphic illustration of the transport of cholesterol in Sertoli cells.

The role of cholesterol metabolism in spermatogenesis. The role of cholesterol in sperm production and male fertility is well established. The process of spermatogenesis consists of a sequence of differentiation and proliferative phases, which are further divided into spermatogenic, meiotic and mitotic stages. Each stage involves different cell types, including spermatids, spermatocytes and spermatogonia (26,27). Additionally, increases in lipid droplets are observed throughout spermatogenesis (28), demonstrating an intimate association between lipid metabolism alterations and fertility during spermatogenesis. Steroidogenesis also requires large amounts of cholesterol (29), while in the seminiferous tubules, cholesterol is required for the differentiation of germinal cells to spermatozoids (gametogenesis/spermatogenesis) (30). Considerable evidence also indicates that, in males and females, cholesterol is required for the development of fertility and gametes. In male mice, the absence of the 24-dehydrocholesterol reductase gene, which encodes a cholesterol biosynthetic enzyme, induces infertility (31). Therefore, cholesterol is required for the mass production of germ cells during spermatogenesis. The plasma membranes of sperm are also heavily loaded with cholesterol when sperm are released from the seminiferous epithelium. Previously, experiments that determined the role of cholesterol de novo synthesis identified that the incorporation rate of ${ }^{14} \mathrm{C}$ acetate into cholesterol was increased during the development of pachytene, leptotene and zygotene stages (32). This indicates that de novo synthesis of cholesterol is increased in these germ cells, which was associated with increased diameter and surface area of germ cells. In late pachynema, the rate of cholesterol synthesis tends to decrease and remains low throughout the following phases of spermatogenesis, including sperm maturation. In contrast to cholesterol, the incorporation capacity of acetate into dolichol was enhanced in round spermatids and late pachytene spermatocytes, and later diminished and remained low in mature sperm cells (32). These observations indicate that the preliminary phases of cholesterol synthesis in round spermatids and pachytene spermatocytes may precede dolichol synthesis, which acts as a critical constituent in the production of membrane glycoproteins. However, the detailed role of cholesterol metabolism within germ cells has not been identified clearly, which is primarily due to the complex connections between germ cells and the supporting Sertoli cells.

Proteins responsible for the regulation of cholesterol metabolism in Sertoli cells

$A B C A 1$. ABCA1, a member of the ABC transporter superfamily, transfers phospholipids and cholesterol out of the peripheral cells to lipid-free apolipoprotein A1, which results in the formation of pre- $\beta$ HDL and is the rate-limiting step in the process of HDL biosynthesis $(33,34)$. Genetic mutations in ABCA1 lead to the absence of plasma HDL, the accumulation of $\mathrm{CE}$ in various body tissues and an increased incidence of atherosclerosis (AS), which are collectively termed Tangier disease (35). By contrast, ABCA1 overexpression in mice enhances plasma HDL levels and prevents the development AS $(36,37)$. However, research has demonstrated that, although ABCA1 in macrophages has an important role in preventing atherosclerosis, it has limited influence on HDL levels in the plasma. In addition, in mice, liver ABCA1 serves as a predominant factor to determine plasma HDL levels $(38,39)$. ABCA1 is highly expressed in the testes $(40,41)$, indicating that ABCA1 may also be involved in the regulation of testicular lipid transport, which is largely segregated from the peripheral circulation (42). Selva et al (3) observed that, in the seminiferous tubule, Sertoli cells account for the majority of the ABCA1 expression. Consistent with the high ABCA1 expression levels, Sertoli cells that express ABCA1 exhibit substantial cholesterol efflux to lipid-free apolipoprotein A1. Conversely, ABCA1-deficient Sertoli cells exhibited decreased cholesterol efflux and accumulation of oil red O-positive lipid droplets, effects that were reversed by ABCA1 restoration, demonstrating that the cholesterol efflux from cultured Sertoli cells is mediated by ABCA1. Fig. 2 is a schematic representation of the regulation of cholesterol content in Sertoli cells. Importantly, in apolipoprotein $\mathrm{A}^{-1-}$ mice, Sertoli cells do not exhibit excess lipids, indicating that deficiency of plasma HDL is insufficient to lead to lipid accumulation in Sertoli cells and that ABCA1 has a direct role in modulating Sertoli cell lipid efflux (43). Furthermore, $\mathrm{ABCA}^{-/-}$mice exhibit reduced testosterone levels and fewer spermatozoa compared with wild-type (WT) mice, indicating that ABCA1 deficiency compromises the Sertoli cell functions and male fertility, but does not lead to complete sterility (3). A deeper understanding of the roles of ABCA1 in lipid transport in spermatogenesis requires further investigation.

SR-BI. SR-BI, which belongs to the SR superfamily, is an important integral membrane glycoprotein on the cell 


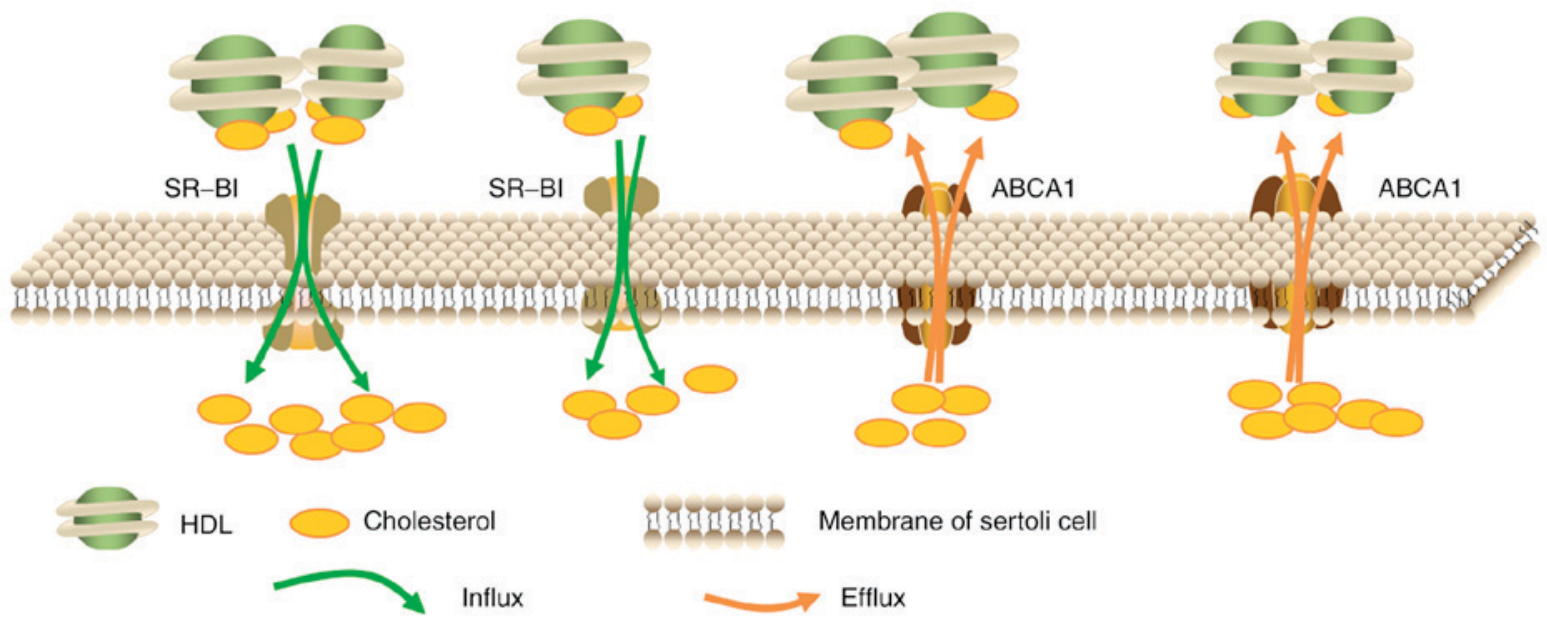

Figure 1. The transport of cholesterol in Sertoli cells. Generally, ABCA1 and SR-BI, two important cholesterol transporters on the membrane, mediate the transport of cholesterol in Sertoli cells. Sertoli cells obtain a large amount of cholesterol from the uptake of HDL-cholesterol esters through SR-BI. In addition, ABCA1 mediates the efflux of surplus cholesterol in Sertoli cells, which maintains the homeostasis of cholesterol levels. ABCA1, ATP-binding cassette subfamily A member 1; SR-BI, scavenger receptor class B member I; HDL, high-density lipoprotein.

membrane. Structurally,SR-BI and CD36 share $30 \%$ homology in amino acid sequence. Functionally, it is a physiological receptor for HDL that facilitates the uptake of HDL-CE, a process that is termed RCT (44). Unlike LDL receptors, which internalize whole lipoprotein particles, SR-BI selectively uptakes cholesterol from HDL into cells. In the testes, the highest expression of SR-BI is observed in steroidogenic Leydig cells $(45,46)$, and the remaining expression occurs in Sertoli cells (46). Previously, Shiratsuchi et al (47) demonstrated that the activity of phosphatidylserine (PS)-mediated phagocytosis of spermatogenic cells by Sertoli cells was enhanced by SR-BI upregulation and inhibited by an anti-SR-BI antibody, indicating that SR-BI acts as a PS receptor, enabling Sertoli cells to recognize and phagocytose spermatogenic cells. Furthermore, Rigotti et al (45) demonstrated that in the testes, a large proportion of lipoprotein-derived cholesterol used for steroidogenesis is acquired through SR-BI (45). Akpovi et al (20) observed that, in mink testes, the overexpression of SR-BI is associated with increased esterified cholesterol levels in Sertoli cells. In addition, Casado et al (48) reported that hormone-sensitive lipase knockout mice exhibited upregulated SR-BI expression, increased phosphorylated (p)-extracellular signal-regulated kinase, p-Akt and p-SRC proto-oncogene levels, and lipid accumulation in Sertoli cells, indicating that SR-BI may be involved in the uptake of $\mathrm{CE}$ for spermatogenesis and steroidogenesis in the testes.

$L X R \alpha / \beta . L X R \alpha / \beta$ have important roles in the maintenance of cellular cholesterol homeostasis. The tissue distribution of these two LXR isoforms is different. LXR $\beta$ is expressed ubiquitously throughout the body, while LXR $\alpha$ is predominantly distributed in the kidney, liver and intestine $(49,50)$. Oxysterols, which are the oxidized derivatives of cholesterol, are the endogenous ligands for LXRs. In addition, T0901317 and GW3965 are synthetic specific ligands. Upon activation, LXRs form heterodimers with RXRs, which are capable of activating the transcription of genes involved in cholesterol efflux. Previously established LXR target genes include ABCA1/G1/G5/G8 (51-53), apolipoprotein A1, apolipoprotein E $(54,55)$ and SR-BI (56). Robertson et al (25) demonstrated that from as early as 2.5 months, cholesterol began to accumulate in the Sertoli cells of LXR $\beta^{-/-}$mice. At around 10 months, although Sertoli cells structure remained comparatively intact and spermatocytes and spermatogonia were still detectable, large and numerous droplets, in addition to the absence of mature germ cells, were observed. By 20 months, lipid accumulation was highest and few cell types were observed. Genetic data from whole testis indicated that the LXR $\beta$ was the predominant transcript in the testis (25). Furthermore, Annicotte et al (57) reported that LXR $\beta$ was present specifically in the Sertoli cells and seminiferous tubules as early as 16.5 days post-conception in embryos, which further highlights the importance of LXR $\beta$ in these cells. Furthermore, results also indicated that LXR $\alpha$ cannot be detected with in situ hybridization (57), and that $\mathrm{LXR}^{-1-}$ mice did not exhibit lipid accumulation and exhibited almost undisturbed spermatogenesis, indicating that the presence of LXR $\beta$ is enough to maintain cholesterol homeostasis in the testis. More importantly, in the $\mathrm{LXR} \alpha^{-1 /} / \beta^{-/}$mouse, the testicular phenotype is more destructive compared with that observed in the LXR $\beta^{-1-}$ mouse. Furthermore, it was also demonstrated that WT animals that were fed a diet mixed with the synthetic LXR agonist T0901317 exhibited increased levels of the LXR target genes sterol regulatory element-binding protein-1c (SREBP-1c) and ABCG1 in their testes $(53,58)$, which was supported by results in MSC-1 cells treated with T0901317 where ABCA1 levels were increased (51). LXR deficiency may also cause dysfunction in these regulatory elements in Sertoli cells. However, the accurate molecular mechanisms underlying the regulation of cholesterol homeostasis by LXR in Sertoli cells and male fertility require further investigation.

$R X R \alpha / \beta / \gamma$. RXR $\alpha / \beta / \gamma$ belongs to the vertebrate nuclear receptor superfamily (59). In rats, $R X R \alpha / \beta$ transcripts are distributed widely throughout the body, whereas RXR $\gamma$ is only expressed in certain types of tissues. It has been demonstrated that $\mathrm{RXR} \beta$ is necessary for normal spermatogenesis in rats, 


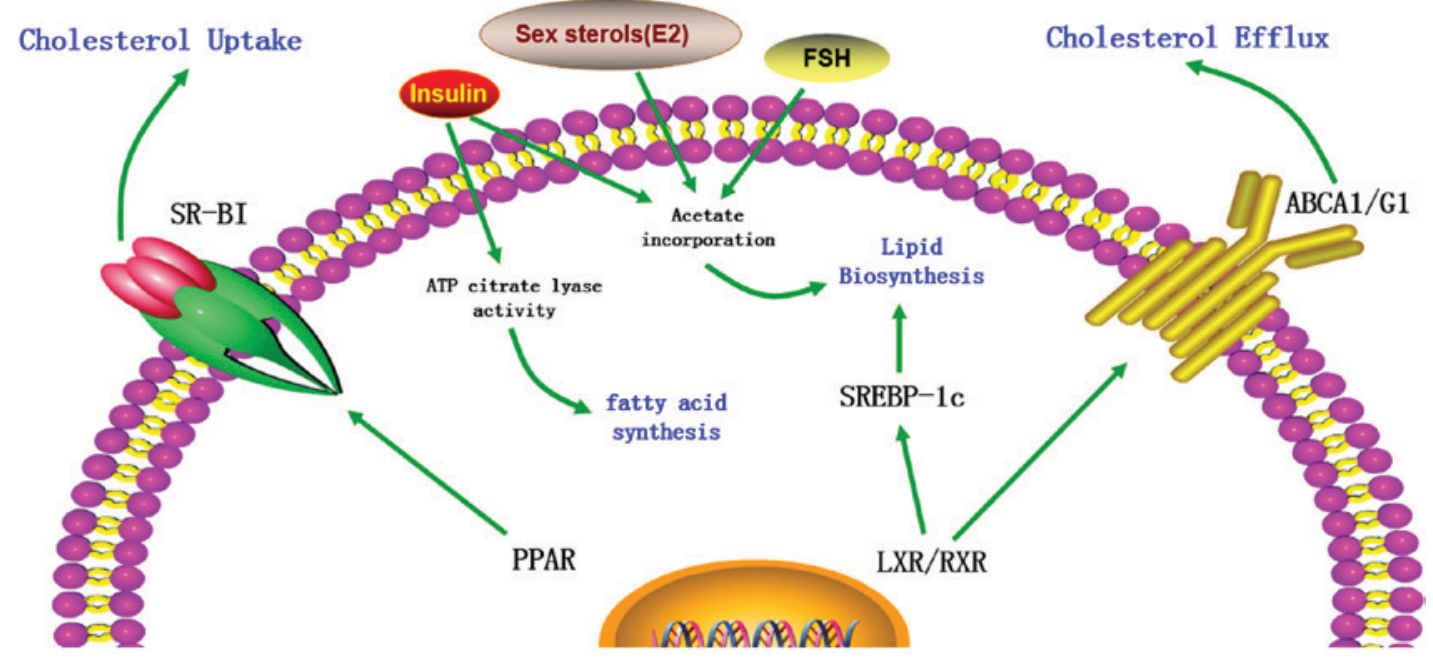

Figure 2. The regulation of cholesterol content in Sertoli cells. The cholesterol metabolism in Sertoli cells is regulated by various factors, including transporters, transcription factors and hormones. LXR/RXR heterodimers increase the cellular cholesterol content by enhancing the levels of SREBP-1c and decrease cholesterol levels by increasing ABCA1 expression. In addition, cholesterol levels are increased by PPAR via increased expression of SR-BI. Furthermore, FSH, insulin and certain sex sterols, including 17 $\beta$-estradiol (E2), increase the cholesterol content in Sertoli cells by increasing acetate incorporation. Insulin also increases the levels of cholesterol and fatty acids by stimulating the activity of ATP citrate lyase. LXR, liver X receptor; RXR, retinoid X receptor; SREBP-1c, sterol regulatory element-binding protein-1c; ABCA1, ATP-binding cassette subfamily A member 1; PPAR, peroxisome proliferator-activated receptor; SR-BI, scavenger receptor class B member I; FSH, follicle-stimulating hormone; ATP, adenosine triphosphate; E2, 17ß-estradiol (E2).

and $\operatorname{RXR} \beta^{-/-}$males exhibit abnormalities in spermiogenesis and spermiation. The biological functions of Sertoli cells may be severely affected by abnormal RXR $\beta$ expression, which is supported by various observations. In the testes of WT mice, only Sertoli cells express RXR $\beta$. In addition, in RXR $\beta^{-/}$mice, Sertoli cell abnormalities precede the presence of abnormal spermatids by at least a week. In 29-day-old mutants, prior to the completion of the first spermatogenic cycle, lipid droplets were observed in Sertoli cells (49). Therefore, the earliest lipid accumulation detected in Sertoli cells cannot due to the phagocytosis of spermatids remnants. Furthermore, in the testes of retinoic acid receptor (RAR) $\alpha^{-/-}$mice and not RAR $\beta^{-/}$mice, although sperm release was blocked during phagocytosis of retained spermatids, lipid droplets in Sertoli cells were not observed (60), indicating that lipid accumulation cannot be attributed to the impaired spermiation. Combined, the results indicate that the lipid deposition observed in the Sertoli cells of RXR $\beta^{-/}$mice reflects a complex metabolic disorder. As Sertoli cells are critical for spermatid maturation, the obstacles of spermiation and spermiogenesis in $\mathrm{RXR} \beta^{--}$mutant testes may due to compromised function of Sertoli cells. Furthermore, as $\mathrm{RXR} \beta$ may serve as a heterodimeric partner for peroxisome proliferator-activated receptor (PPAR) $\beta$, which is expressed abundantly in Sertoli cells (61), compromised PPAR function may also contribute to the lipid deposition in $\mathrm{RXR}^{-/-}$mutant Sertoli cells (62). However, the role of RXR in the lipid metabolism of Sertoli cells requires further investigation.

FSH and insulin. FSH, which is produced and secreted by the anterior pituitary gland, is a crucial reproduction factor in mammals. In females, FSH facilitates the maturation of follicles and the production of estrogen, while in males it enhances Sertoli cell proliferation in the immature testis and mature spermatogenesis $(63,64)$. The levels of FSH are regulated by hormones released by gonadotropin through estrogen feedback and the hypothalamic pituitary gonadal axis (65). Guma et al (66) demonstrated that FSH and insulin promoted the lipid metabolism of Sertoli cells by augmenting acetate incorporation into Sertoli cell lipids. These stimulatory activities of FSH and insulin do not involve protein synthesis, indicating a potential effect via modulation of enzyme activity or glucose transport in Sertoli cells. In addition, treatment with insulin, but not FSH, also enhanced the ATP citrate lyase activity, indicating the biological relevance of insulin on the fatty acid synthesis of Sertoli cells. Oliveira et al (67) demonstrated that insulin signaling is indispensable for Sertoli cell lipid metabolism, as the absence of insulin transforms Sertoli cell metabolism from glycolysis to the Krebs cycle, which inhibits the development of germ cells.

Sex sterols and PPAR $\alpha / \beta / \delta / \gamma$. Sex steroids are also involved in the modulation of cholesterol metabolism in Sertoli cells. The effects of $5 \alpha$-dihydrotestosterone on Sertoli cell cholesterol metabolism resembled those of insulin, but were less obvious. By contrast, 17 $\beta$-estradiol (E2) promotes increases in acetate concentration by amplifying the transcript levels of acetyl-CoA hydrolase, therefore contributing to the production of by-products that are essential for the maintenance of cholesterol synthesis in Sertoli cells (68). In mature Sertoli cells, 18-carbon polyunsaturated fatty acids (PUFAs) are efficiently converted into 22- and 24-carbon PUFAs with the assistance of certain metabolic enzymes, including fatty acid elongases, and $\Delta 5$ and $\Delta 6$ desaturases (69). However, hormonal dysregulation may disrupt PUFA synthesis, as Sertoli cells treated with testosterone exhibited reduced activities of the $\Delta 5$ and $\Delta 6$ desaturases, therefore repressing the steps involving $\Delta 5$ and $\Delta 6$ desaturases (70). These effects are important as inhibition of $\Delta 5$ and $\Delta 6$ desaturases activity is likely to restrict the incorporative process of long chain PUFAs into sperm membranes, which may ultimately decrease sperm 


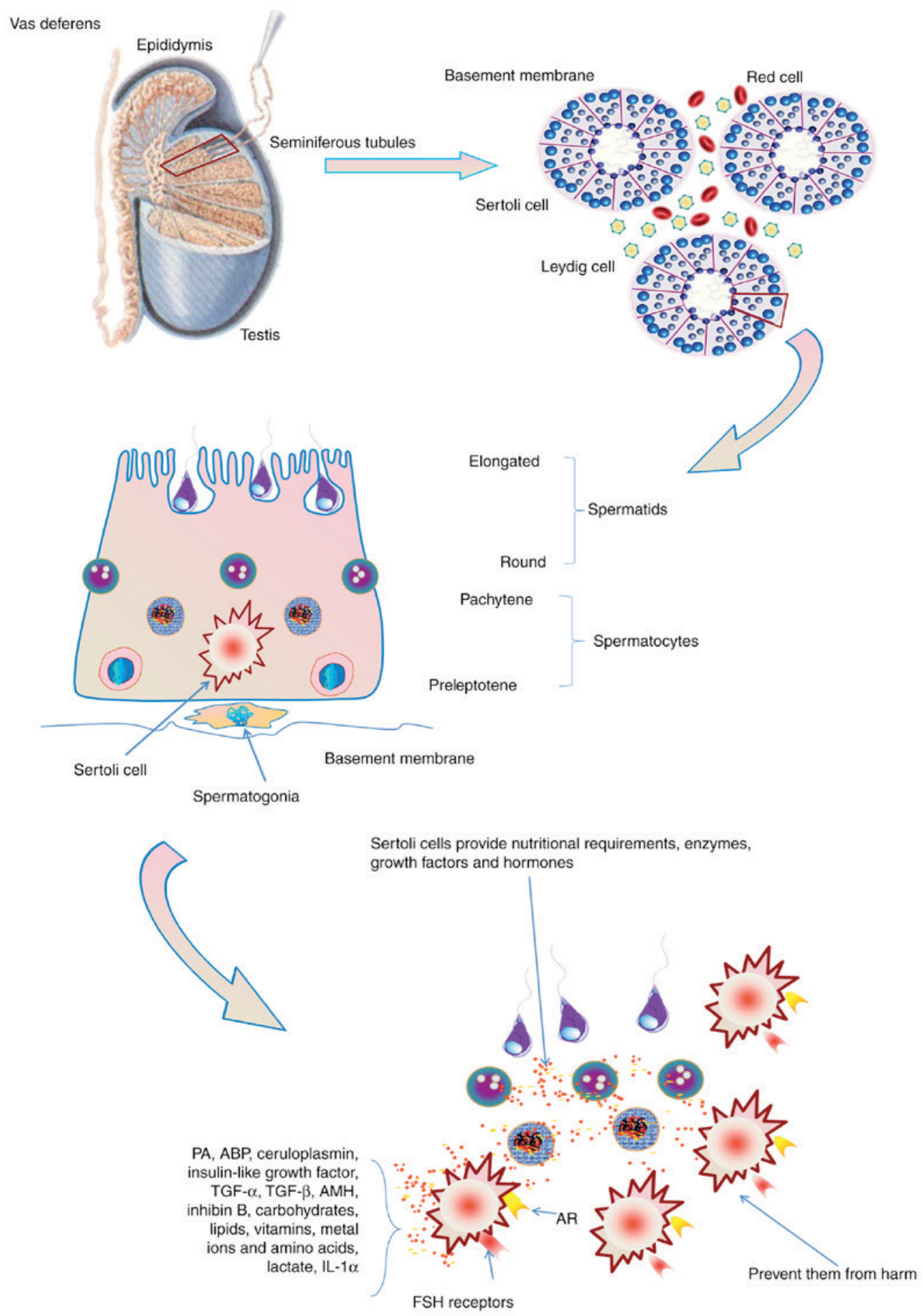

Figure 3. The role of Sertoli cells in spermatogenesis. Sertoli cells provide the nutritional requirements of germ cells, including carbohydrates, lipids, vitamins, metal ions and amino acids in spermatogenesis. Furthermore, a functional Sertoli cell produces various types of enzymes, growth factors and hormones, and prevent germ cells from harm. Sertoli cells also express the AR and receptors for FSH. AR, androgen receptor; FSH, follicle-stimulating hormone; PA, plasminogen activator; ABP, androgen-binding protein; TGF, transforming growth factor; AMH, anti-Müllerian hormone; IL, interleukin.

membrane flexibility and fluidity. PUFAs possess several double bonds in their backbone, which, with an increased number of lipids, improves the fluidity and flexibility of sperm membranes (71).

In addition, lipid oxidation in Sertoli cells is also regulated by PPAR $\alpha / \beta / \delta / \gamma$, which serve as sensors and derivatives of fatty acids and thus influence the lipid and cholesterol metabolic pathways. Regueira et al (72) demonstrated that the activation of PPAR $\alpha$ and PPAR $\beta / \delta$ augmented the expression of the cholesterol transporter SR-BI in Sertoli cells, therefore promoting the selective uptake of CE. Furthermore, PPAR activation enhanced acetyl-CoA carboxylase phosphorylation, resulting in reduced enzyme activity, which promotes the incorporation of fatty acyl-CoA into the mitochondria for further oxidation, and increased long and medium chain dehydrogenase enzyme and L-carnitine palmitoyl transferase 
1 mRNA levels (72). These results indicate that PPAR $\alpha$ and $\operatorname{PPAR} \beta / \delta$ are indispensable for cholesterol metabolism and lipid oxidation in Sertoli cells, while the upstream factors regulated by the PPAR system remain uncertain.

\section{Conclusions and perspectives}

Sertoli cells are essential for spermatogenesis as they provide developing germ cells with physical support and a source of growth factors, hormones, nutrients and energy. The regulation of Sertoli cell metabolism has received attention from numerous reproductive biologists as it may be involved in the fate of germ cells. In fact, the maintenance of spermatogenesis primarily relies on the metabolic collaboration between Sertoli and germ cells, which is shown as a schematic representation in Fig. 3. It involves various metabolic pathways and a network of signal transduction. Various factors regulate metabolic activity in Sertoli cells, which are primary targets for hormonal signaling. Any alterations in the metabolic behavior of Sertoli cells may cause abnormalities in spermatogenesis and ultimately lead to male fertility. Thus, Sertoli cells metabolism has a key role in the regulation of spermatogenesis.

Currently, the source of cholesterol utilized for Sertoli cell metabolism and spermatogenesis is not well-established, which is largely because the process is complex and suitable in vitro systems are scarce. Sertoli cells obtain a small portion of cholesterol from the de novo synthesis pathway, whereas the majority of cholesterol is obtained from lipoprotein particles in the interstitial compartment, as described above. The uncertainty may be resolved by thoroughly checking the phenotypes of conditional cell knockouts of genes that are implicated in cholesterol synthesis and transport in various types of testicular cells. In this respect, such a model has not previously been developed. Certain studies have examined mouse models with large-scale inactivation of genes responsible for cholesterol transport, with the results indicating that cholesterol transport is essential for normal spermatogenesis. For example, mice with overall knockout of type 2 apolipoprotein E receptor (73) and mice with partial knockout of apolipoprotein B (74) exhibited severely abnormal fertility. In addition, further cholesterol-associated genes and proteins are also implicated in male fertility, as demonstrated by different mouse models (51-56). Furthermore, negative effects of overload of dietary cholesterol on fertility in mice further demonstrated the importance of cholesterol transport in sperm development $(75,76)$. The examination of conditional knockouts of cytochrome P450 family 51 in the testis indicated that de novo cholesterol synthesis has an important role in spermatogenesis. Detecting the phenotypes of the testis and fertility in these knockout models provides important insights into the importance of cholesterol synthesis and transport in germ and Sertoli cells, as well as in male fertility.

Additionally, certain developed methods, including high-resolution lipid imaging in vivo or in vitro, may be instrumental in understanding the process of cholesterol metabolism in Sertoli cells (77). For example, Gimpl and Gehrig-Burger (78) reported that fluorescent and photoreactive sterol probes reflect the movement and location of these lipids, in addition to the cross-talk with proteins. Although these novel methods have not been largely applied to the investigation of Sertoli cell metabolism, they hold huge promise for understanding cholesterol and lipid metabolism in Sertoli cells during spermatogenesis, and may also help to explain previously unexplained male infertility cases, which may be caused by perturbations of cholesterol metabolism in the testes due to various environmental or genetic factors.

\section{Acknowledgements}

The present review was supported by grants from the Natural Sciences Foundation of Hunan Province (grant nos. 14JJ2084 and 2015JJ2128), the Zhengxiang Scholar (Xiangyang Tang) Program of the University of South China, The Construct Program of the Key Discipline in Hunan Province (Basic Medicine Sciences in University of South China) and Hunan Province Cooperative Innovation Center for Molecular Target New Drug Study (2015-351).

\section{References}

1. Bhasin S: Approach to the infertile man. J Clin Endocrinol Metab 92: 1995-2004, 2007.

2. Holstein AF, Schulze W and Davidoff M: Understanding spermatogenesis is a prerequisite for treatment. Reprod Biol Endocrinol 1: 107, 2003.

3. Selva DM, Hirsch-Reinshagen V, Burgess B, Zhou S, Chan J, McIsaac S, Hayden MR, Hammond GL, Vogl AW and Wellington CL: The ATP-binding cassette transporter 1 mediates lipid efflux from Sertoli cells and influences male fertility. J Lipid Res 45: 1040-1050, 2004.

4. Chase TH, Lyons BL, Bronson RT, Foreman O, Donahue LR, Burzenski LM, Gott B, Lane P, Harris B, Ceglarek U, et al: The mouse mutation 'thrombocytopenia and cardiomyopathy' (trac) disrupts Abcg5: A spontaneous single gene model for human hereditary phytosterolemia/sitosterolemia. Blood 115: 1267-1276, 2010.

5. Huang LS, Voyiaziakis E, Markenson DF, Sokol KA, Hayek T and Breslow JL: apo B gene knockout in mice results in embryonic lethality in homozygotes and neural tube defects, male infertility, and reduced HDL cholesterol ester and apo A-I transport rates in heterozygotes. The Journal of clinical investigation 96: 2152-2161, 1995

6. Snouwaert JN, Brigman KK, Latour AM, Malouf NN, Boucher RC, Smithies O and Koller BH: An animal model for cystic fibrosis made by gene targeting. Science 257: 1083-1088, 1992.

7. Blendy JA, Kaestner KH, Weinbauer GF, Nieschlag E and Schütz G: Severe impairment of spermatogenesis in mice lacking the CREM gene. Nature 380: 162-165, 1996.

8. Fofana M, Maboundou JC, Bocquet J and Le Goff D: Transfer of cholesterol between high density lipoproteins and cultured rat Sertoli cells. Biochem Cell Biol 74: 681-686, 1996.

9. Umehara T, Kawashima I, Kawai T, Hoshino Y, Morohashi KI, Shima Y, Zeng W, Richards JS and Shimada M: Neuregulin 1 regulates proliferation of Leydig cells to support spermatogenesis and sexual behavior in adult mice. Endocrinology 157: 4899-4913, 2016

10. Andersen JM and Dietschy JM: Relative importance of high and low density lipoproteins in the regulation of cholesterol synthesis in the adrenal gland, ovary, and testis of the rat. J Biol Chem 253: 9024-9032, 1978.

11. Steinberger E, Root A, Ficher M and Smith KD: The role of androgens in the initiation of spermatogenesis in man. J Clin Endocrinol Metab 37: 746-751, 1973

12. McLachlan RI, Wreford NG, O'Donnell L, de Kretser DM and Robertson DM: The endocrine regulation of spermatogenesis: Independent roles for testosterone and FSH. J Endocrinol 148: $1-9,1996$

13. Boussouar $\mathrm{F}$ and Benahmed M: Lactate and energy metabolism in male germ cells. Trends Endocrinol Metab 15: 345-350, 2004. 
14. Grass J and Hauser ER: The influence of early age mastectomy and unilateral ovariectomy on reproductive performance of the bovine. J Anim Sci 53: 171-176, 1981

15. Grootegoed JA, Oonk RB, Jansen R and van der Molen HJ: Metabolism of radiolabelled energy-yielding substrates by rat Sertoli cells. J Reprod Fertil 77: 109-118, 1986.

16. Robinson R and Fritz IB: Metabolism of glucose by Sertoli cells in culture. Biol Reprod 24: 1032-1041, 1981.

17. Mruk DD and Cheng CY: Sertoli-Sertoli and Sertoli-germ cell interactions and their significance in germ cell movement in the seminiferous epithelium during spermatogenesis. Endocr Rev 25: 747-806, 2004.

18. Petersen C and Soder O: The sertoli cell--a hormonal target and 'super' nurse for germ cells that determines testicular size. Horm Res 66: 153-161, 2006.

19. Wiebe JP and Tilbe KS: De novo synthesis of steroids (from acetate) by isolated rat Sertoli cells. Biochem Biophys Res Commun 89: 1107-1113, 1979.

20. Akpovi CD, Yoon SR, Vitale ML and Pelletier RM: The predominance of one of the SR-BI isoforms is associated with increased esterified cholesterol levels not apoptosis in mink testis. J Lipid Res 47: 2233-2247, 2006.

21. Fofana M, Travert C, Carreau S and Le Goff D: Evaluation of cholesteryl ester transfer in the seminiferous tubule cells of immature rats in vivo and in vitro. J Reprod Fertil 118: 79-83, 2000.

22. Nakanishi Y and Shiratsuchi A: Phagocytic removal of apoptotic spermatogenic cells by Sertoli cells: Mechanisms and consequences. Biol Pharm Bull 27: 13-16, 2004.

23. Pelletier RM: The blood-testis barrier: The junctional permeability, the proteins and the lipids. Prog Histochem Cytochem 46 49-127, 2011

24. Nebel A, Flachsbart F, Till A, Caliebe A, Blanché H, Arlt A, Häsler R, Jacobs G, Kleindorp R, Franke A, et al: A functional EXO1 promoter variant is associated with prolonged life expectancy in centenarians. Mech Ageing Dev 130: 691-699, 2009.

25. Robertson KM, Schuster GU, Steffensen KR, Hovatta O, Meaney S, Hultenby K, Johansson LC, Svechnikov K, Söder O and Gustafsson JA: The liver X receptor-\{beta\} is essential for maintaining cholesterol homeostasis in the testis. Endocrinology 146: 2519-2530, 2005.

26. Eddy EM: Regulation of gene expression during spermatogenesis Semin Cell Dev Biol 9: 451-457, 1998

27. Hodgson YM, Irby DC, Kerr JB and de Kretser DM: Studies of the structure and function of the Sertoli cell in a seasonally breeding rodent. Biol Reprod 21: 1091-1098, 1979.

28. Osuga J, Ishibashi S, Oka T, Yagyu H, Tozawa R, Fujimoto A, Shionoiri F, Yahagi N, Kraemer FB, Tsutsumi O and Yamada N: Targeted disruption of hormone-sensitive lipase results in male sterility and adipocyte hypertrophy, but not in obesity. Proc Natl Acad Sci USA 97: 787-792, 2000.

29. Gwynne JT and Strauss JF III: The role of lipoproteins in steroidogenesis and cholesterol metabolism in steroidogenic glands. Endocr Rev 3: 299-329, 1982.

30. Kabbaj O, Yoon SR, Holm C, Rose J, Vitale ML and Pelletier RM: Relationship of the hormone-sensitive lipase-mediated modulation of cholesterol metabolism in individual compartments of the testis to serum pituitary hormone and testosterone concentrations in a seasonal breeder, the mink (Mustela vison). Biol Reprod 68 722-734, 2003

31. Wechsler A, Brafman A, Shafir M, Heverin M, Gottlieb H, Damari G, Gozlan-Kelner S, Spivak I, Moshkin O, Fridman E, et al: Generation of viable cholesterol-free mice. Science 302: 2087, 2003.

32. Potter JE, Millette CF, James MJ and Kandutsch AA: Elevated cholesterol and dolichol synthesis in mouse pachytene spermatocytes. J Biol Chem 256: 7150-7154, 1981.

33. Brewer HB Jr and Santamarina-Fojo S: Clinical significance of high-density lipoproteins and the development of atherosclerosis: Focus on the role of the adenosine triphosphate-binding cassette protein A1 transporter. Am J Cardiol 92: 10K-6K, 2003.

34. Hayden MR, Clee SM, Brooks-Wilson A, Genest J Jr, Attie A and Kastelein JJ: Cholesterol efflux regulatory protein, Tangier disease and familial high-density lipoprotein deficiency. Curr Opin Lipidol 11: 117-122, 2000.

35. Singaraja RR, Brunham LR, Visscher H, Kastelein JJ and Hayden MR: Efflux and atherosclerosis: The clinical and biochemical impact of variations in the ABCA1 gene. Arterioscler Thromb Vasc Biol 23: 1322-1332, 2003.
36. Singaraja RR, Bocher V, James ER, Clee SM, Zhang LH, Leavitt BR, Tan B, Brooks-Wilson A, Kwok A, Bissada N, et al: Human ABCA1 BAC transgenic mice show increased high density lipoprotein cholesterol and ApoAI-dependent efflux stimulated by an internal promoter containing liver $\mathrm{X}$ receptor response elements in intron 1. J Biol Chem 276: 33969-33979, 2001.

37. Singaraja RR, Fievet C, Castro G, James ER, Hennuyer N, Clee SM, Bissada N, Choy JC, Fruchart JC, McManus BM, et al: Increased ABCA1 activity protects against atherosclerosis. J Clin Invest 110: 35-42, 2002.

38. Haghpassand M, Bourassa PA, Francone OL and Aiello RJ: Monocyte/macrophage expression of ABCA1 has minimal contribution to plasma HDL levels. J Clin Invest 108: 1315-1320, 2001.

39. Aiello RJ, Brees D, Bourassa PA, Royer L, Lindsey S, Coskran T, Haghpassand $\mathrm{M}$ and Francone OL: Increased atherosclerosis in hyperlipidemic mice with inactivation of ABCA1 in macrophages. Arterioscler Thromb Vasc Biol 22: 630-637, 2002.

40. Wellington CL, Walker EK, Suarez A, Kwok A, Bissada N, Singaraja R, Yang YZ, Zhang LH, James E, Wilson JE, et al: ABCA1 mRNA and protein distribution patterns predict multiple different roles and levels of regulation. Lab Invest 82: 273-283, 2002.

41. Lawn RM, Wade DP, Couse TL and Wilcox JN: Localization of human ATP-binding cassette transporter 1 (ABC1) in normal and atherosclerotic tissues. Arterioscler Thromb Vasc Biol 21: 378-385, 2001.

42. Pelletier RM and Byers SW: The blood-testis barrier and Sertoli cell junctions: Structural considerations. Microsc Res Tech 20: 3-33, 1992.

43. Plump AS, Erickson SK, Weng W, Partin JS, Breslow JL and Williams DL: Apolipoprotein A-I is required for cholesteryl ester accumulation in steroidogenic cells and for normal adrenal steroid production. J Clin Invest 97: 2660-2671, 1996.

44. Di Pietro N, Formoso G and Pandolfi A: Physiology and pathophysiology of oxLDL uptake by vascular wall cells in atherosclerosis. Vascul Pharmacol 84: 1-7, 2016.

45. Rigotti A, Miettinen HE and Krieger M: The role of the high-density lipoprotein receptor SR-BI in the lipid metabolism of endocrine and other tissues. Endocr Rev 24: 357-387, 2003.

46. Landschulz KT, Pathak RK, Rigotti A, Krieger M and Hobbs HH: Regulation of scavenger receptor, class B, type I, a high density lipoprotein receptor, in liver and steroidogenic tissues of the rat. J Clin Invest 98: 984-995, 1996.

47. Shiratsuchi A, Kawasaki Y, Ikemoto M, Arai H and Nakanishi Y: Role of class B scavenger receptor type I in phagocytosis of apoptotic rat spermatogenic cells by Sertoli cells. J Biol Chem 274: 5901-5908, 1999.

48. Casado ME, Huerta L, Ortiz AI, Pérez-Crespo M, Gutiérrez-Adán A, Kraemer FB, Lasunción MÁ, Busto R and Martín-Hidalgo A: HSL-knockout mouse testis exhibits class B scavenger receptor upregulation and disrupted lipid raft microdomains. J Lipid Res 53: 2586-2597, 2012.

49. Seol W, Choi HS and Moore DD: Isolation of proteins that interact specifically with the retinoid $X$ receptor: Two novel orphan receptors. Mol Endocrinol 9: 72-85, 1995.

50. Willy PJ, Umesono K, Ong ES, Evans RM, Heyman RA and Mangelsdorf DJ: LXR, a nuclear receptor that defines a distinct retinoid response pathway. Genes Dev 9: 1033-1045, 1995.

51. Costet P, Luo Y, Wang N and Tall AR: Sterol-dependent transactivation of the $\mathrm{ABC} 1$ promoter by the liver $\mathrm{X}$ receptor/retinoid $\mathrm{X}$ receptor. J Biol Chem 275: 28240-28245, 2000.

52. Repa JJ, Turley SD, Lobaccaro JA, Medina J, Li L, Lustig K, Shan B, Heyman RA, Dietschy JM and Mangelsdorf DJ: Regulation of absorption and ABC1-mediated efflux of cholesterol by RXR heterodimers. Science 289: 1524-1529, 2000.

53. Kennedy MA, Venkateswaran A, Tarr PT, Xenarios I, Kudoh J, Shimizu N and Edwards PA: Characterization of the human ABCG1 gene: Liver X receptor activates an internal promoter that produces a novel transcript encoding an alternative form of the protein. J Biol Chem 276: 39438-39447, 2001.

54. Fayard E, Schoonjans K and Auwerx J: Xol INXS: Role of the liver $\mathrm{X}$ and the farnesol $\mathrm{X}$ receptors. Curr Opin Lipidol 12: $113-120,2001$

55. Oram JF and Vaughan AM: ABCA1-mediated transport of cellular cholesterol and phospholipids to HDL apolipoproteins. Curr Opin Lipidol 11: 253-260, 2000. 
56. Malerød L, Juvet LK, Hanssen-Bauer A, Eskild W and Berg T: Oxysterol-activated LXRalpha/RXR induces hSR-BI-promoter activity in hepatoma cells and preadipocytes. Biochem Biophys Res Commun 299: 916-923, 2000.

57. Annicotte JS, Schoonjans K and Auwerx J: Expression of the liver $\mathrm{X}$ receptor alpha and beta in embryonic and adult mice. Anat Rec A Discov Mol Cell Evol Biol 277: 312-316, 2004.

58. Repa JJ,Liang G,Ou J,Bashmakov Y,Lobaccaro JM,ShimomuraI, Shan B, Brown MS, Goldstein JL and Mangelsdorf DJ: Regulation of mouse sterol regulatory element-binding protein-1c gene (SREBP-1c) by oxysterol receptors, LXRalpha and LXRbeta. Genes Dev 14: 2819-2830, 2000.

59. Evans RM and Mangelsdorf DJ: Nuclear Receptors, RXR, and the Big Bang. Cell 157: 255-266, 2014.

60. Lohnes D, Mark M, Mendelsohn C, Dollé P, Dierich A, Gorry P, Gansmuller A and Chambon P: Function of the retinoic acid receptors (RARs) during development (I). Craniofacial and skeletal abnormalities in RAR double mutants. Development 120 2723-2748, 1994.

61. Braissant O, Foufelle F, Scotto C, Dauca M and Wahli W: Differential expression of peroxisome proliferator-activated receptors (PPARs): Tissue distribution of PPAR-alpha, -beta, and -gamma in the adult rat. Endocrinology 137: 354-366, 1996.

62. Kastner P, Mark M, Leid M, Gansmuller A, Chin W, Grondona JM, Décimo D, Krezel W, Dierich A and Chambon P: Abnormal spermatogenesis in RXR beta mutant mice. Genes Dev 10: 80-92, 1996.

63. Macklon NS and Fauser BC: Follicle development during the normal menstrual cycle. Maturitas 30: 181-188, 1998.

64. Plant TM and Marshall GR: The functional significance of FSH in spermatogenesis and the control of its secretion in male primates. Endocr Rev 22: 764-786, 2001.

65. Midgley Ar J and Jaffe RB: Regulation of human gonadotropins: 4. Correlation of serum concentration $\mathrm{s}$ of follicle stimulating and luteinizing hormones during the menstrual cycle. J Clin Endocrinol 28: 1699-1703, 1999.

66. Guma FC, Wagner M, Martini LH and Bernard EA: Effect of FSH and insulin on lipogenesis in cultures of Sertoli cells from immature rats. Braz J Med Biol Res 30: 591-597, 1997.

67. Oliveira PF, Alves MG, Rato L, Laurentino S, Silva J, Sá R, Barros A, Sousa M, Carvalho RA, Cavaco JE and Socorro S: Effect of insulin deprivation on metabolism and metabolism-associated gene transcript levels of in vitro cultured human Sertoli cells Biochim Biophys Acta 1820: 84-89, 2012.
68. Oliveira PF, Alves MG, Rato L, Silva J, Sá R, Barros A, Sousa M, Carvalho RA, Cavaco JE and Socorro S: Influence of $5 \alpha$-dihydrotestosterone and $17 \beta$-estradiol on human Sertoli cells metabolism. Int J Androl 34: e612-e620, 2011.

69. Saether T, Tran TN, Rootwelt H, Christophersen BO and Haugen TB: Expression and regulation of delta5-desaturase, delta6-desaturase, stearoyl-coenzyme A (CoA) desaturase 1, and stearoyl-CoA desaturase 2 in rat testis. Biol Reprod 69: 117-124, 2003.

70. Carosa E, Radico C, Giansante N, Rossi S, D'Adamo F, Di Stasi SM, Lenzi A and Jannini EA: Ontogenetic profile and thyroid hormone regulation of type- 1 and type- 8 glucose transporters in rat Sertoli cells. Int J Androl 28: 99-106, 2005.

71. Israelachvili JN, Marcelja S and Horn RG: Physical principles of membrane organization. Q Rev Biophys 13: 121-200, 1980.

72. Regueira M, Riera MF, Galardo MN, Pellizzari EH, Cigorraga SB and Meroni SB: Activation of PPAR $\alpha$ and PPAR $\beta / \delta$ regulates Sertoli cell metabolism. Mol Cell Endocrinol 382: 271-281, 2014.

73. Manova K and Bachvarova RF: Expression of c-kit encoded at the $\mathrm{W}$ locus of mice in developing embryonic germ cells and presumptive melanoblasts. Dev Biol 146: 312-324, 1991.

74. Orth JM, Jester WF Jr and Qiu J: Gonocytes in testes of neonatal rats express the c-kit gene. Mol Reprod Dev 45: 123-131, 1996.

75. Rossi P, Albanesi C, Grimaldi P and Geremia R: Expression of the mRNA for the ligand of c-kit in mouse Sertoli cells. Biochem Biophys Res Commun 176: 910-914, 1991.

76. Mauduit C, Hamamah S and Benahmed M: Stem cell factor/c-kit system in spermatogenesis. Hum Reprod Update 5: 535-545, 1999.

77. Dias TR, Rato L, Martins AD, Simões VL, Jesus TT, Alves MG and Oliveira PF: Insulin deprivation decreases caspase-dependent apoptotic signaling in cultured rat sertoli cells. ISRN Urol 2013: 970370, 2013

78. Gimpl G and Gehrig BK: Probes for studying cholesterol binding and cell biology. Steroids 76: 216-231, 2011.

This work is licensed under a Creative Commons Attribution-NonCommercial-NoDerivatives 4.0 International (CC BY-NC-ND 4.0) License, 